\title{
Production of $X(3872)$ at high multiplicity
}

\author{
Eric Braaten, ${ }^{*}$ Li-Ping He๑, ${ }^{\dagger}$ and Kevin Ingles $\odot^{*}$ \\ Department of Physics, The Ohio State University, Columbus, Ohio 43210, USA \\ Jun Jiang $\mathbb{1}^{\S}$ \\ School of Physics, Shandong University, Jinan, Shandong 250100, China
}

(Received 29 December 2020; accepted 16 February 2021; published 16 April 2021)

\begin{abstract}
The dependence of the production of the $X(3872)$ meson on the hadron multiplicity in $p p$ collisions has been used as evidence against $X$ being a charm-meson molecule. The argument is based in part on the incorrect assumption that the cross section for the breakup of $X$ by scattering with comovers can be approximated by a geometric cross section inversely proportional to the binding energy of $X$. The breakup cross section should instead be approximated by the probability-weighted sum of the cross sections for the scattering of comoving pions from the charm-meson constituents of $X$, which is insensitive to the binding energy. A simple modification of the comover interaction model gives excellent fits to the data from the LHCb Collaboration on the multiplicity dependence of the production of $X$ and $\psi(2 S)$ using parameters compatible with $X$ being a loosely bound charm-meson molecule.
\end{abstract}

DOI: 10.1103/PhysRevD.103.L071901

\section{INTRODUCTION}

Since the unexpected discovery of the $X(3872)$ meson [also known as $\chi_{c 1}(3872)$ ] in 2003 [1], dozens of other exotic heavy hadrons not predicted by the quark model have been discovered [2-5]. They present a major challenge to our understanding of QCD. The nature of $X(3872)$ ( $X$ for short) is a particularly important issue, because it remains the exotic heavy hadron for which the most detailed experimental information is available. The $X$ was discovered in the decay mode $J / \psi \pi^{+} \pi^{-}$, and it has since been observed in six other decay modes. Its quantum numbers were determined in 2013 to be $J^{P C}=1^{++}$[6]. The LHCb Collaboration recently made the most precise measurements of its mass $M_{X}$ and the first measurements of its decay width $[7,8]$. The difference between $M_{X}$ and the $D^{* 0} \bar{D}^{0}$ threshold is $\varepsilon_{X}=-0.07 \pm 0.12 \mathrm{MeV}$. This implies an upper bound on the binding energy of $X:\left|\varepsilon_{X}\right|<0.22 \mathrm{MeV}$ at $90 \%$ confidence level.

The information $J^{P C}=1^{++}$and $\left|\varepsilon_{X}\right|<0.22 \mathrm{MeV}$ is sufficient to conclude that $X$ must be a loosely bound $S$-wave molecule with the particle content $\left(D^{* 0} \bar{D}^{0}+D^{0} \bar{D}^{* 0}\right) / \sqrt{2}$

\footnotetext{
*braaten.1@osu.edu

he.1011@buckeyemail.osu.edu

*ingles.27@buckeyemail.osu.edu

§jiangjun87@sdu.edu.cn
}

Published by the American Physical Society under the terms of the Creative Commons Attribution 4.0 International license. Further distribution of this work must maintain attribution to the author(s) and the published article's title, journal citation, and DOI. Funded by SCOAP. and with universal properties determined by $\varepsilon_{X}$ [9]. The mean separation of its constituents is $r_{X}=\left(8 \mu\left|\varepsilon_{X}\right|\right)^{-1 / 2}$, where $\mu$ is the reduced mass of $D^{* 0} \bar{D}^{0}$. The upper bound $\left|\varepsilon_{X}\right|<0.22 \mathrm{MeV}$ implies $r_{X}>4.8 \mathrm{fm}$. Thus, this amazing hadron has a radius more than an order of magnitude larger than that of ordinary hadrons. More relevant to the other exotic heavy hadrons is what $X$ would have been if not for the fine-tuning of its mass to the $D^{* 0} \bar{D}^{0}$ threshold. The possibilities that have been proposed include the $\mathrm{P}$-wave charmonium state $\chi_{c 1}(2 P)$, an isospin-0 charm-meson molecule, and an isospin-1 compact tetraquark. In all these cases, the tuning of the mass to the $D^{* 0} \bar{D}^{0}$ threshold produces resonant couplings to $D^{* 0} \bar{D}^{0}$ and $D^{0} \bar{D}^{* 0}$ that transforms $X$ into a loosely bound molecule of neutral charm mesons.

Shortly after the discovery of $X$ in $B$-meson decays [1], its existence was confirmed in $p \bar{p}$ collisions [10]. The production of $X$ at a hadron collider can be resolved into two contributions: prompt production by strong interactions at the primary collision vertex and the b-decay contribution from weak decays of hadrons containing a bottom quark or antiquark at a displaced secondary vertex. The behavior of these two contributions may provide evidence for the nature of $X$. One significant difference is the hadronic environment in which $X$ is embedded. In the decay of a $b$ hadron, at most a few additional hadrons emerge from the secondary vertex. In prompt production at the LHC, hundreds of additional hadrons may emerge from the primary vertex. Collisions with comoving hadrons could break $X$ up into its charm-meson constituents and thus decrease its prompt cross section. 
The LHCb Collaboration has studied the dependence on the hadron multiplicity of the production of $X$ in $p p$ collisions at the center-of-mass energy $\sqrt{s}=8 \mathrm{TeV}$ [11]. The charmonium state $\psi(2 S)$ ( $\psi^{\prime}$ for short) provides a convenient benchmark, because it also decays into $J / \psi \pi^{+} \pi^{-}$, and its mass is close to $M_{X}$. The LHCb Collaboration measured the ratio of the prompt production rates for $X$ and $\psi^{\prime}$ in the $J / \psi \pi^{+} \pi^{-}$decay channel as functions of the number $N_{\text {tracks }}$ of tracks in the vertex detector. The prompt $X$-to- $\psi^{\prime}$ ratio decreases significantly with increasing $N_{\text {tracks }}$.

Esposito et al. have used the comover interaction (CI) model to calculate the dependence of the prompt $X$-to- $\psi^{\prime}$ ratio on the charged-particle multiplicity $N_{\mathrm{ch}}$ [12]. Their result for $X$ as a compact tetraquark is consistent with the LHCb data, while their result for $X$ as a molecule with a geometric cross section decreases much too rapidly with $N_{\text {ch }}$. They concluded that the LHCb data support $X$ being a tetraquark and strongly disfavor it being a molecule. Their results if $X$ is a molecule were based in part on the incorrect assumption that its breakup reaction rate can be approximated by the geometric cross section $\pi r_{X}^{2}$, which is proportional to $1 /\left|\varepsilon_{X}\right|$. It should instead be approximated by the cross section for scattering from the charm-meson constituents of $X$, which is insensitive to $\varepsilon_{X}$. We show below that a simple modification of the CI model provides excellent fits to the LHCb data on the multiplicity dependence of $X$ and $\psi^{\prime}$ production with parameters compatible with $X$ being a loosely bound charm-meson molecule.

\section{COMOVER INTERACTION MODEL}

The CI model was developed to describe the suppression of charmonium states in relativistic $p$-nucleus and nucleusnucleus collisions by taking into account final-state interactions with comoving hadrons created by the collision [13-15]. Ferreiro used the CI model [16] to describe the suppression of $\psi^{\prime}$ relative to $J / \psi$ in $d-\mathrm{Au}$ and $p-\mathrm{Pb}$ collisions at RHIC [17-19]. Ferreiro and Lansberg developed a more elaborate version of the CI model [20] to describe the suppression of $\Upsilon(2 S)$ and $\Upsilon(3 S)$ relative to $\Upsilon$ in $p-\mathrm{Pb}$ collisions at LHC [21,22]. A modified version of their model was applied by Esposito et al. to the production of $X$ in $p p$ collisions [12].

In the CI model, the survival probability of a $c \bar{c}$ or $b \bar{b}$ meson $\mathcal{Q}$ in $p p$ collisions is [23]

$$
S_{\mathcal{Q}}=\exp \left(-\frac{\left\langle v \sigma_{\mathcal{Q}}\right\rangle d N / d y}{\sigma_{p p}} \log \frac{d N / d y}{N_{p p}}\right),
$$

where $d N / d y$ is the light-hadron multiplicity per unit range of rapidity and $\left\langle v \sigma_{\mathcal{Q}}\right\rangle$ is the reaction rate for the breakup of $\mathcal{Q}$ averaged over comovers. The nondiffractive cross section $\sigma_{p p}(s)$ depends on the center-of-mass energy $\sqrt{s}$, while $N_{p p}(s, y)$ may also depend on the rapidity $y . N_{p p}$ is the multiplicity below which the effects of comovers are negligible: $S_{\mathcal{Q}}=1$, if $d N / d y<N_{p p}$. The estimates for $\sigma_{p p}$ in Ref. [12] are $63 \mathrm{mb}$ at $\sqrt{s}=7 \mathrm{TeV}$ and $70 \mathrm{mb}$ at $13 \mathrm{TeV}$. A logarithmic interpolation in $s$ gives $\sigma_{p p}=65 \mathrm{mb}$ at $\sqrt{s}=8 \mathrm{TeV}$. The range of pseudorapidity for the LHCb spectrometer is $2.0<\eta<4.8$. An estimate of $N_{p p}$ in that region can be obtained by multiplying the mean chargedparticle multiplicity for the LHCb detector [24] by $3 / 2$ to take into account neutral particles and then dividing by $\Delta y=2.8$, which gives $N_{p p} \approx 6$.

In the CI model, the comovers are usually assumed to be either pions with mass $m_{\pi} \approx 140 \mathrm{MeV}$ or massless gluons. In Ref. [20], the momentum distribution of the comovers in the $\mathcal{Q}$ rest frame was assumed to be a Bose-Einstein distribution in the two-dimensional transverse plane with an effective temperature $T_{\text {eff. }}$. In Ref. [12], it was assumed to be a three-dimensional Bose-Einstein distribution. Reference [20] introduced a simplistic model for the breakup cross section $\sigma_{\mathcal{Q}}$ as a function of the comover energy $E_{\pi}: \pi r_{\mathcal{Q}}^{2}\left(1-E_{\mathcal{Q}}^{\mathrm{thr}} / E_{\pi}\right)^{n}$, where $E_{\mathcal{Q}}^{\mathrm{thr}}$ is the threshold energy for the breakup of $\mathcal{Q}$. In Ref. [12], that same model was used instead for the breakup reaction rate $v \sigma_{\mathcal{Q}}$. In Ref. [20], $T_{\text {eff }}$ was determined by fitting data on the suppression of $\Upsilon(2 S)$ and $\Upsilon(3 S)$ in $p-\mathrm{Pb}$ and $\mathrm{Pb}-\mathrm{Pb}$ collisions. The fitted value of $T_{\text {eff }}$ is approximately linear in $n$ between $\frac{1}{2}$ and 2, and its extrapolation to $n=0$ is roughly $100 \mathrm{MeV}$. For $n=1$, the effective temperature is $T_{\text {eff }}=(250 \pm 50) \mathrm{MeV}$. These same values of $n$ and $T_{\text {eff }}$ were used in Ref. [12].

\section{ANALYSIS OF REF. [12]}

In Ref. [12], their results for the prompt $X$-to- $\psi^{\prime}$ ratio were compared with preliminary LHCb data [25]. The theoretical results were normalized to the first $\mathrm{LHCb}$ data point at $N_{\text {tracks }}=20$. As shown in Fig. 1, their narrow error

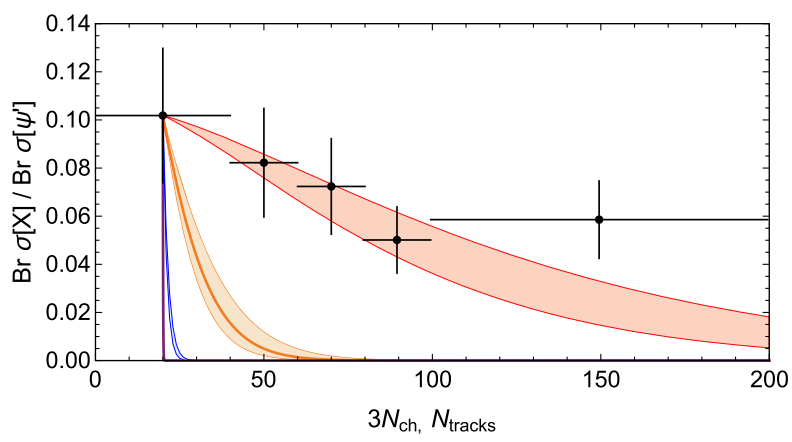

FIG. 1. Prompt $X$-to- $\psi^{\prime}$ ratio as a function of the multiplicity $N_{\text {ch }}$. The preliminary LHCb data from Ref. [25] are shown in bins of $N_{\text {tracks }}$, which is identified with $3 N_{\mathrm{ch}}$. The two higher error bands are for a compact tetraquark from Ref. [12] (red band, near the data) and using their value of $\left\langle v \sigma_{X}\right\rangle$ (orange band). The two lower error bands are for a charm-meson molecule from Ref. [12] (blue band) and using their value of $\left\langle v \sigma_{X}\right\rangle$ (purple band, almost vertical). 
band for a molecule decreases precipitously to almost 0 near $N_{\text {tracks }}=25$, while their error band for a tetraquark gives a good fit to the $\mathrm{LHCb}$ data in the next three bins of $N_{\text {tracks }}$, which extend from 40 to 100.

It is implied in Ref. [12] that their error bands follow from inserting their breakup reaction rates $\left\langle v \sigma_{\mathcal{Q}}\right\rangle$ into the ratio $S_{X} / S_{\psi^{\prime}}$ of the survival probabilities given by Eq. (1). The values of $\left\langle v \sigma_{\mathcal{Q}}\right\rangle$ in Ref. [12] are $5.15 \pm 0.84 \mathrm{mb}$ for $\psi^{\prime}$, $11.61 \pm 1.69 \mathrm{mb}$ for $X$ if it is a tetraquark, and $1197 \pm$ $171 \mathrm{mb}$ for $X$ if it is a molecule with $\left|\varepsilon_{X}\right|=116 \mathrm{keV}$. The prescription used to obtain these values was not specified. The resulting error bands are shown in Fig. 1. The error band using their value of $\left\langle v \sigma_{X}\right\rangle$ if $X$ is a tetraquark decreases almost exponentially to 0 , and it lies well below the LHCb data even in the second bin of $N_{\text {tracks }}$. Thus, the error bands in Ref. [12] must be determined by physics not captured by the survival probability in Eq. (1).

\section{IV. $\pi X$ BREAKUP REACTION RATES}

Cross sections for low-energy $\pi X$ scattering can be calculated using a nonrelativistic effective field theory for charm mesons and pions called XEFT [26]. It provides a systematically improvable description of the sector of QCD consisting of $D^{*} \bar{D}, D \bar{D}^{*}, D \bar{D} \pi$, or $X$ with total energy near the $D^{*} \bar{D}$ threshold [26] and also the sector consisting of $D^{*} \bar{D}^{*}, D^{*} \bar{D} \pi, D \bar{D}^{*} \pi, D \bar{D} \pi \pi$, or $X \pi$ near the $D^{*} \bar{D}^{*}$ threshold [27]. A Galilean-invariant formulation of XEFT that exploits the approximate conservation of mass in the transitions $D^{*} \leftrightarrow D \pi$ was introduced in Ref. [28] and further developed in Ref. [29]. Galilean invariance guarantees that cross sections are the same in all Galilean frames, and it reduces the number of Feynman diagrams by requiring conservation of the total number of $\pi, D^{*}, \bar{D}^{*}$, and $X$ mesons.

The breakup cross section for $\pi^{+} X \rightarrow D^{*+} \bar{D}^{* 0}$ was first calculated in Ref. [27] in the CM frame using original XEFT. The cross sections for $\pi^{+} X \rightarrow D^{*+} \bar{D}^{* 0}$ and $\pi^{0} X \rightarrow$ $D^{* 0} \bar{D}^{* 0}$ are calculated using Galilean-invariant XEFT in Ref. [30]. In Fig. 2, the cross sections are shown as functions of the collision energy $E_{c}$, which is the total kinetic energy in the $\mathrm{CM}$ frame. They have dramatic peaks near their thresholds, with peak values comparable to the geometric cross section $\pi r_{X}^{2}$, which is $1200 \mathrm{mb}$ if $\varepsilon_{X}=116 \mathrm{keV}$. In the limit $\varepsilon_{X} \rightarrow 0$, the cross section for $\pi^{+} X \rightarrow D^{*+} \bar{D}^{* 0}$ approaches a delta function in $E_{c}$ at $\left(\mu_{\pi X} / \mu_{\pi}\right) \delta_{0+}$, where $\delta_{0+}=5.9 \mathrm{MeV}$ is the $D^{*+}$-to- $D^{0} \pi^{+}$ energy difference, and $\mu_{\pi X}$ and $\mu_{\pi}$ are the reduced masses for $\pi X$ and $\pi D$. The energy-weighted integral of the P-wave contribution to the cross section reduces in the limit to

$$
\int d E_{c} E_{c} \sigma\left[\pi^{+} X \rightarrow D^{*+} \bar{D}^{* 0}\right] \rightarrow \frac{2 \pi \sqrt{2} \mu_{\pi X}^{2} \delta_{0+}^{3 / 2} g^{2}}{\mu_{\pi}^{1 / 2}\left(2 \sqrt{m_{\pi}} f_{\pi}\right)^{2}},
$$

where $g /\left(2 \sqrt{m_{\pi}} f_{\pi}\right)$ is the $D^{* 0}$-to- $D^{0} \pi^{0}$ coupling constant. This is the integral required to calculate the contribution to $\left\langle v \sigma_{X}\right\rangle$ from a three-dimensional Bose-Einstein distribution

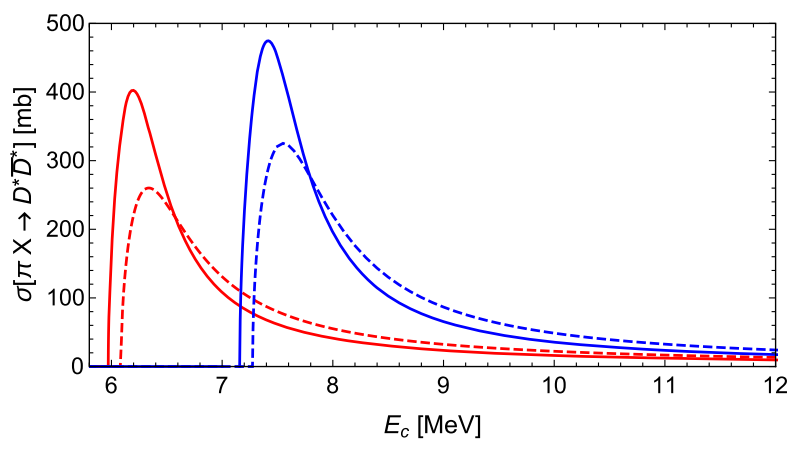

FIG. 2. Breakup cross sections for $\pi^{+} X \rightarrow D^{*+} \bar{D}^{0}$ (red curves with lower threshold) and $\pi^{0} X \rightarrow D^{* 0} \bar{D}^{0}$ (blue curves with higher threshold) as functions of the collision energy. The binding energy of $X$ is $116 \mathrm{keV}$ (solid curves) and $232 \mathrm{keV}$ (dashed curves).

of pions. The corresponding integral for $\pi^{0} X \rightarrow D^{* 0} \bar{D}^{* 0}$ is obtained by replacing $\delta_{0+}$ by the $D^{* 0}$-to- $D^{0} \pi^{0}$ energy difference $\delta_{00}=7.0 \mathrm{MeV}$. Their contribution to $\left\langle v \sigma_{X}\right\rangle$ decreases from 0.2 to 0.04 to $0.02 \mathrm{mb}$ as $T_{\text {eff }}$ increases from 100 to 200 to $300 \mathrm{MeV}$.

When the $\pi X$ collision energy is well above the resonance region in Fig. 2, the pion can scatter off an individual constituent of $X$, and this will necessarily break up the bound state. The constituents of $X$ are $D^{* 0}$ and $\bar{D}^{0}$ with probability $1 / 2$ and $D^{0}$ and $\bar{D}^{* 0}$ with probability $1 / 2$. The total $\pi X$ breakup cross section can be approximated by the weighted sum of $\pi D$ and $\pi D^{*}$ cross sections,

$\sigma^{\text {incl }}[\pi X] \approx \frac{1}{2}\left(\sigma\left[\pi D^{0}\right]+\sigma\left[\pi \bar{D}^{0}\right]+\sigma\left[\pi D^{* 0}\right]+\sigma\left[\pi \bar{D}^{* 0}\right]\right)$.

A sufficient condition for the validity of this approximation is that $E_{c}$ is well above the resonance region shown in Fig. 2.

For nonrelativistic collision energies, the largest cross sections are those allowed in Galilean-invariant XEFT. The specific final states from $\pi X$ scattering taken into account by Eq. (3) are $D^{*} \bar{D} \pi$ and $D \bar{D}^{*} \pi$ with at least one neutral charm meson. The cross sections for $\pi D^{0} \rightarrow \pi D$ and $\pi D^{* 0} \rightarrow \pi D^{*}$ are calculated in Ref. [30]. In the region $\delta_{00} \ll E_{c} \ll m_{\pi}$, they are approximately constant. The total P-wave contribution to the $\pi X$ breakup cross section using Eq. (3) is

$$
\sigma^{\mathrm{incl}}[\pi X] \approx \frac{4 \mu_{\pi}^{2}\left(\mu_{\pi}^{2}+\mu_{\pi *}^{2}\right) g^{4}}{\pi\left(2 \sqrt{m_{\pi}} f_{\pi}\right)^{4}},
$$

where $\mu_{\pi *}$ is the $\pi D^{*}$ reduced mass. An overestimate of the contribution of this region to $\left\langle v \sigma_{X}\right\rangle$ can be obtained by integrating over the range $\delta_{00}<E_{c}<m_{\pi}$. This estimate decreases from 0.05 to 0.02 to $0.01 \mathrm{mb}$ as $T_{\text {eff }}$ increases from 100 to 200 to $300 \mathrm{MeV}$. 
For relativistic collision energies of order $m_{\pi}$ and larger, $\mathrm{XEFT}$ is not applicable. There have been previous calculations of the reaction rates $\langle v \sigma\rangle$ for $\pi X \rightarrow D^{*} \bar{D}^{*}$ in a thermal gas of hadrons. In Ref. [31], the reaction rate decreases from 0.5 to $0.2 \mathrm{mb}$ as the temperature $T$ increases from 100 to $200 \mathrm{MeV}$. In Ref. [32], the reaction rate is approximately constant near $35 \mathrm{mb}$ for $T$ between 100 and $180 \mathrm{MeV}$. There have been previous calculations of the $\pi D$ and $\pi D^{*}$ reaction rates $\langle v \sigma\rangle$ in a thermal gas of hadrons. In Ref. [33], the $\pi D$ and $\pi D^{*}$ reaction rates from pion interactions were calculated at temperatures $T$ from 116 to $156 \mathrm{MeV}$. The reaction rates are approximately independent of $T$, but they depend on a cutoff parameter $\Lambda$. As $\Lambda$ increases from 0.5 to 1.0 to $1.5 \mathrm{GeV}$, the reaction rate from $\pi D \rightarrow \pi D$ increases from 2 to 6 to $10 \mathrm{mb}$. The reaction rate from $\pi D^{*} \rightarrow \pi D^{*}$ is much smaller, increasing from 0.007 to 0.04 to $0.1 \mathrm{mb}$. In Ref. [34], the structure of hadrons was taken into account by using a form factor with cutoff momentum $\Lambda$. For $\Lambda=\infty$, the estimate of $\left\langle v \sigma_{X}\right\rangle$ using Eq. (3) increases from 25 to $37 \mathrm{mb}$ as $T$ increases from 100 to $200 \mathrm{MeV}$, while for $\Lambda=1 \mathrm{GeV},\left\langle v \sigma_{X}\right\rangle \approx 15 \mathrm{mb}$ almost independent of $T$.

\section{ANALYSIS OF LHCB DATA}

The LHCb data in Ref. [11] consist of the prompt fractions for both $X$ and $\psi^{\prime}$ and the $X$-to- $\psi^{\prime}$ ratios for both prompt and $b$-decay production in Fig. 3 . The horizontal bars attached to each data point indicate the bin of the variable $N_{\text {tracks }}$, and the value of $N_{\text {tracks }}$ for the data point is at the multiplicityweighted center of the bin. Since the pseudorapidity range of the $\mathrm{LHCb}$ vertex detector is $1.6<\eta<4.9$ [35], the multiplicity $d N / d y$ can be approximated by $\frac{3}{2}\left(N_{\text {tracks }} / 3.3\right)$. The prompt fraction $f_{\text {prompt }}$ for $\psi^{\prime}$ is about $87 \%$ in the first bin of $N_{\text {tracks. }}$. It first decreases as $N_{\text {tracks }}$ increases, but then it appears to level off at about $70 \%$. This behavior is incompatible with the assumption that the prompt cross section is proportional to the survival probability $S_{\psi^{\prime}}$ given by Eq. (1). That assumption requires $f_{\text {prompt }}$ to decrease almost exponentially to 0 as $N_{\text {tracks }}$ increases.

A possible interpretation of the $\mathrm{LHCb}$ data on the prompt $\psi^{\prime}$ fraction in Fig. 3 is that the prompt cross section has two

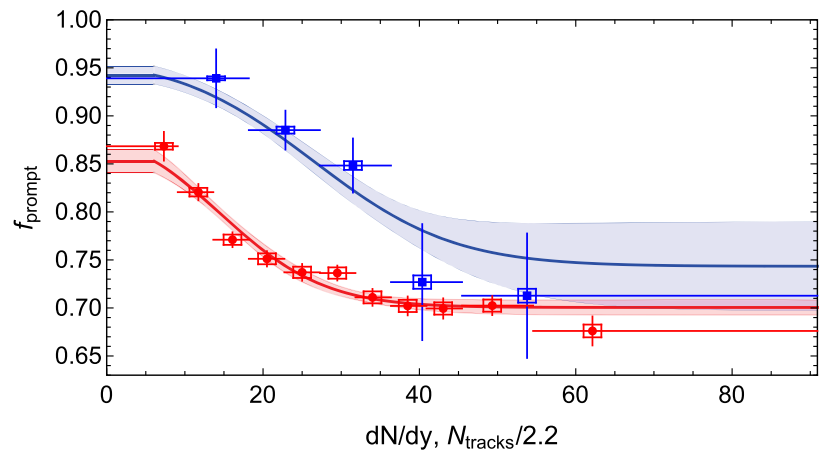

components: one independent of $d N / d y$ and the other proportional to $S_{\psi^{\prime}}$. The two components could arise from the phase-space structure of the $p p$ collisions. Prompt $\psi^{\prime \prime}$ 's created at a space-time point and with a momentum that puts them out of reach of most of the comoving pions give a contribution to the cross section that does not depend on $d N / d y$. The remaining prompt $\psi$ 's are broken up with the probability $1-S_{\psi^{\prime}}$, so their contribution to the prompt cross section is proportional to $S_{\psi^{\prime}}$.

This interpretation motivates a simple modification of the CI model. We denote the fraction of the prompt $\mathcal{Q}$ mesons out of reach of comoving pions by $f_{\text {out }, \mathcal{Q}}$ and their contribution to the prompt cross section by $\sigma_{\text {out, } \mathcal{Q}}$. The prompt cross section can be expressed as

$$
\sigma_{\text {prompt }}[\mathcal{Q}]=\left[1+\left(1 / f_{\text {out }, \mathcal{Q}}-1\right) S_{\mathcal{Q}}\right] \sigma_{\text {out }, \mathcal{Q}},
$$

which depends on $d N / d y$ through $S_{\mathcal{Q}}$. We assume the $b$-decay cross section $\sigma_{b \text { decay, } \mathcal{Q}}$ does not depend on $d N / d y$. The prompt fraction for $\mathcal{Q}$ is

$$
f_{\text {prompt }}[\mathcal{Q}]=\frac{1+\left(1 / f_{\text {out }, \mathcal{Q}}-1\right) S_{\mathcal{Q}}}{1+1 / F_{\text {out }, \mathcal{Q}}+\left(1 / f_{\text {out }, \mathcal{Q}}-1\right) S_{\mathcal{Q}}},
$$

where $F_{\text {out }, \mathcal{Q}}=\sigma_{\text {out }, \mathcal{Q}} / \sigma_{b \text { decay }, \mathcal{Q}}$. The prompt $X$-to- $\psi^{\prime}$ ratio is

$$
\frac{\operatorname{Br} \sigma_{\text {prompt }}[X]}{\operatorname{Br} \sigma_{\text {prompt }}\left[\psi^{\prime}\right]}=N_{X / \psi^{\prime}} \frac{1+\left(1 / f_{\text {out }, X}-1\right) S_{X}}{1+\left(1 / f_{\text {out }, \psi^{\prime}}-1\right) S_{\psi^{\prime}}},
$$

where $N_{X / \psi^{\prime}}$ is the product of $\sigma_{\mathrm{out}, X} / \sigma_{\mathrm{out}, \psi^{\prime}}$ and the ratio of the branching fractions into $J / \psi \pi^{+} \pi^{-}$. The $b$-decay $X$-to- $\psi^{\prime}$ ratio is

$$
\frac{\operatorname{Br} \sigma_{b \text { decay }}[X]}{\operatorname{Br} \sigma_{b \text { decay }}\left[\psi^{\prime}\right]}=N_{X / \psi^{\prime}} \frac{F_{\text {out }, \psi^{\prime}}}{F_{\text {out }, X}}
$$

We have carried out a global fit to the LHCb data by minimizing the $\chi^{2}$ for the 26 data points in Fig. 3 with respect to the five adjustable parameters in Eqs. (6)-(8) and

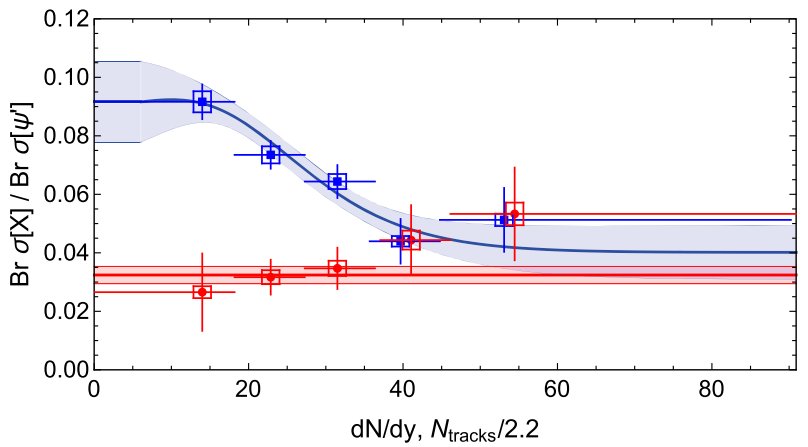

FIG. 3. Prompt fractions (left panel) and $X$-to- $\psi^{\prime}$ production ratios (right panel) as functions of the multiplicity $d N / d y$. The LHCb data in Ref. [11] for the prompt $X$ fraction (blue squares), the prompt $\psi^{\prime}$ fraction (red dots), the prompt $X$-to- $\psi^{\prime}$ ratio (blue squares) and the $b$-decay $X$-to- $\psi \psi^{\prime}$ ratio (red dots) are shown in bins of $N_{\text {tracks }} / 2.2$. The curves and their error bands are from a global fit to the LHCb data. 
the two breakup reaction rates $\left\langle v \sigma_{X}\right\rangle$ and $\left\langle v \sigma_{\psi^{\prime}}\right\rangle$. Each data point is interpreted as the value at the multiplicity-weighted center of the bin, and its statistical and correlated errors are added in quadrature. The resulting fits are shown in Fig. 3. The error bands correspond to an increase of $\chi^{2}$ by less than 1. The quality of the fits is very good with $\chi^{2} /$ dof $=0.99$. The fit to the $b$-decay $X$-to- $\psi^{\prime}$ ratio could be improved by adding a parameter that allows $\sigma_{\text {out, }, \psi^{\prime}}$ or $\sigma_{b \text { decay }, X}$ to increase linearly with $d N / d y$. The values of the seven fitted parameters are $f_{\text {out }, \psi^{\prime}}=0.40 \pm 0.03, f_{\text {out }, X}=$ $0.18 \pm 0.04, \quad F_{\text {out }, \psi^{\prime}}=2.3 \pm 0.1, \quad F_{\text {out }, X}=2.9 \pm 0.7$, $\left\langle v \sigma_{\psi^{\prime}}\right\rangle=3.9 \pm 0.8 \mathrm{mb},\left\langle v \sigma_{X}\right\rangle=2.6 \pm 0.7 \mathrm{mb}$, and $N_{X / \psi^{\prime}}=$ $0.04 \pm 0.01$. The correlation matrix for these seven parameters is

$$
\left(\begin{array}{ccccccc}
1 & -0.40 & -0.59 & 0 & +0.83 & -0.28 & +0.35 \\
& 1 & 0 & -0.22 & -0.45 & +0.86 & -0.86 \\
& & 1 & -0.08 & -0.76 & 0 & +0.04 \\
& & & 1 & 0 & -0.29 & -0.20 \\
& & & & 1 & -0.40 & +0.43 \\
& & & & & 1 & -0.83 \\
& & & & & & 1
\end{array}\right) .
$$

The fitted value of $\left\langle v \sigma_{\psi^{\prime}}\right\rangle$ is about $1 \sigma$ smaller than the value in Ref. [12]. The fitted value of $\left\langle v \sigma_{X}\right\rangle$ is about $5 \sigma$ smaller than the value in Ref. [12] if $X$ is a tetraquark. It is about 4 times larger than the contribution from $\pi X \rightarrow$ $D^{*} \bar{D}^{*}$ in a thermal gas of hadrons with $T=100 \mathrm{MeV}$ in Ref. [31]. The fitted value of $\left\langle v \sigma_{X}\right\rangle$ is less than $1 / 4$ the total breakup reaction rate from Ref. [34]. This could be attributed to a failure of the three-dimensional BoseEinstein distribution as a model for comoving pions. A momentum distribution that is isotropic in the two transverse dimensions and the longitudinal dimension seems implausible.

\section{OUTLOOK}

The LHCb data on the multiplicity dependence of the production of $X$ and $\psi^{\prime}$ in $p p$ collisions is incompatible with the assumption that the prompt cross section is proportional to the survival probability in Eq. (1). However, as shown in Fig. 3, a good global fit can be obtained by adding the assumption that some fraction $f_{\text {out }, \mathcal{Q}}$ of the prompt $\mathcal{Q}$ cross section is out of reach of comoving pions. A microscopic description of $p p$ collisions in which these fractions could be calculated would be useful.

The quantum numbers $J^{P C}=1^{++}$and the upper bound $\left|\varepsilon_{X}\right|<0.22 \mathrm{MeV}$ imply that $X$ must be a loosely bound
$\mathrm{S}$-wave molecule of neutral charm mesons with universal properties determined by $\varepsilon_{X}$. Universality is a double-edged sword. It allows definite statements about some properties of $X$, such as $r_{X}$ and the $\pi X$ breakup reaction rate, but it also makes them insensitive to what $X$ would have been if not for the fine-tuning of its mass to the $D^{* 0} \bar{D}^{0}$ threshold. $X$ could have been a more compact charmonium or molecule or tetraquark, but it is transformed into a large neutral-charmmeson molecule by its resonant interactions with $D^{* 0} \bar{D}^{0}$ and $D^{0} \bar{D}^{* 0}$. Given the upper bound on $\left|\varepsilon_{X}\right|$, a model for $X$ as a compact hadron should be interpreted as a fictitious hadron that does not couple to the charm mesons at the nearby $D^{* 0} \bar{D}^{0}$ threshold. It may be an interesting exercise to rule out such a possibility using experimental data, but it is already excluded by theoretical considerations.

The universal physics of a loosely bound S-wave molecule reveals a dramatic failure of the simplistic model in Refs. [12] and [20] for $\left\langle v \sigma_{\mathcal{Q}}\right\rangle$ based on the geometric cross section $\pi r_{\mathcal{Q}}^{2}$. That model overestimates $\left\langle v \sigma_{X}\right\rangle$ by orders of magnitude. The breakup cross section $\sigma_{X}$ is comparable to $\pi r_{X}^{2}$, which is proportional to $1 /\left|\varepsilon_{X}\right|$, only at energies very close to the threshold as shown in Fig. 2. At higher energies, $\sigma_{X}$ is determined by the cross sections for scattering from the constituents of $X$ in Eq. (3), so $\left\langle v \sigma_{X}\right\rangle$ is insensitive to $\varepsilon_{X}$.

Our fit to the LHCb data in Fig. 3 may be a step towards a quantitative understanding of the production of $X$ in highenergy hadron collisions. In a hadron collision, once $X$ is broken up into charm mesons by the collision with a comoving pion, the probability that one of the charm mesons will encounter another charm meson and that they will coalesce into $X$ is extremely small. An attempt to calculate the coalescence contribution to the production in $p p$ collisions of $X$ if it is a molecule was made in Ref. [12]. Coalescence can be much more important in $p$-nucleus and nucleus-nucleus collisions, because the number of charm meson that are created is much larger. The first observation of the production of $X$ in heavy-ion collisions by the CMS Collaboration indicated that the prompt $X$-to- $\psi \psi^{\prime}$ ratio may be much larger in $\mathrm{Pb}-\mathrm{Pb}$ collisions than in $p p$ collisions [36]. Understanding the production of $X$ in $p$-nucleus and nucleus-nucleus collisions even at the qualitative level remains a challenging open problem.

\section{ACKNOWLEDGMENTS}

This work was supported in part by the U.S. Department of Energy under Grant No. DE-SC0011726, the National Natural Science Foundation of China under Grant No. 11905112, and the Natural Science Foundation of Shandong Province under Grant No. ZR2019QA012. L.-P. H. was supported in part by a Presidential Fellowship from Ohio State University. We acknowledge useful communications with A. Esposito and A. Pilloni. 
[1] S. K. Choi et al. (Belle Collaboration), Observation of a Narrow Charmonium-Like State in Exclusive $B^{ \pm} \rightarrow$ $K^{ \pm} \pi^{+} \pi^{-} J / \psi$ decays, Phys. Rev. Lett. 91, 262001 (2003).

[2] A. Ali, J. S. Lange, and S. Stone, Exotics: Heavy pentaquarks and tetraquarks, Prog. Part. Nucl. Phys. 97, 123 (2017).

[3] S. L. Olsen, T. Skwarnicki, and D. Zieminska, Nonstandard heavy mesons and baryons: Experimental evidence, Rev. Mod. Phys. 90, 015003 (2018).

[4] M. Karliner, J. L. Rosner, and T. Skwarnicki, Multiquark States, Annu. Rev. Nucl. Part. Sci. 68, 17 (2018).

[5] N. Brambilla, S. Eidelman, C. Hanhart, A. Nefediev, C. P. Shen, C. E. Thomas, A. Vairo, and C.Z. Yuan, The $X Y Z$ states: Experimental and theoretical status and perspectives, Phys. Rep. 873, 1 (2020).

[6] R. Aaij et al. (LHCb Collaboration), Determination of the $X(3872)$ Meson Quantum Numbers, Phys. Rev. Lett. 110, 222001 (2013).

[7] R. Aaij et al. (LHCb Collaboration), Study of the $\psi_{2}(3823)$ and $\chi_{c 1}(3872)$ states in $B^{+} \rightarrow\left(J \psi \pi^{+} \pi^{-}\right) K^{+}$decays, J. High Energy Phys. 08 (2020) 123.

[8] R. Aaij et al. (LHCb Collaboration), Study of the lineshape of the $\chi_{c 1}(3872)$ state, Phys. Rev. D 102, 092005 (2020).

[9] E. Braaten and M. Kusunoki, Low-energy universality and the new charmonium resonance at $3870 \mathrm{MeV}$, Phys. Rev. D 69, 074005 (2004).

[10] D. Acosta et al. (CDF Collaboration), Observation of the Narrow State $X(3872) \rightarrow J / \psi \pi^{+} \pi^{-}$in $\bar{p} p$ Collisions at $\sqrt{s}=1.96 \mathrm{TeV}$, Phys. Rev. Lett. 93, 072001 (2004).

[11] R. Aaij et al. (LHCb Collaboration), Observation of multiplicity-dependent prompt $\chi_{c 1}(3872)$ and $\psi(2 S)$ production in $p p$ collisions, arXiv:2009.06619.

[12] A. Esposito, E. G. Ferreiro, A. Pilloni, A. D. Polosa, and C. A. Salgado, The nature of $X(3872)$ from high-multiplicity $p p$ collisions, arXiv:2006.15044.

[13] A. Capella, A. Kaidalov, A. Kouider Akil, and C. Gerschel, $J / \psi$ and $\psi^{\prime}$ suppression in heavy ion collisions, Phys. Lett. B 393, 431 (1997).

[14] S. Gavin and R. Vogt, Charmonium Suppression by Comover Scattering in $\mathrm{Pb}+\mathrm{Pb}$ Collisions, Phys. Rev. Lett. 78, 1006 (1997).

[15] D. Kharzeev, C. Lourenco, M. Nardi, and H. Satz, A Quantitative analysis of charmonium suppression in nuclear collisions, Z. Phys. C 74, 307 (1997).

[16] E. G. Ferreiro, Excited charmonium suppression in protonnucleus collisions as a consequence of comovers, Phys. Lett. B 749, 98 (2015).

[17] A. Adare et al. (PHENIX Collaboration), Nuclear Modification of $\psi^{\prime}, \chi_{c}$, and $J / \psi$ Production in $d+$ Au Collisions at $\sqrt{s_{N N}}=200 \mathrm{GeV}$, Phys. Rev. Lett. 111, 202301 (2013).

[18] B. B. Abelev et al. (ALICE Collaboration), Suppression of $\psi(2 S)$ production in $\mathrm{p}-\mathrm{Pb}$ collisions at $\sqrt{s_{\mathrm{NN}}}=5.02 \mathrm{TeV}$, J. High Energy Phys. 12 (2014) 073.

[19] R. Arnaldi (ALICE Collaboration), Inclusive $\psi(2 S)$ production in $\mathrm{p}-\mathrm{Pb}$ collisions with ALICE, Nucl. Phys. A931, 628 (2014).
[20] E. G. Ferreiro and J. P. Lansberg, Is bottomonium suppression in proton-nucleus and nucleus-nucleus collisions at LHC energies due to the same effects?, J. High Energy Phys. 10 (2018) 094.

[21] S. Chatrchyan et al. (CMS Collaboration), Event activity dependence of $\Upsilon(n S)$ production in $\sqrt{s_{N N}}=5.02 \mathrm{TeV} \mathrm{pPb}$ and $\sqrt{s}=2.76 \mathrm{TeV}$ pp collisions, J. High Energy Phys. 04 (2014) 103.

[22] M. Aaboud et al. (ATLAS Collaboration), Measurement of quarkonium production in proton-lead and proton-proton collisions at 5.02 TeV with the ATLAS detector, Eur. Phys. J. C 78, 171 (2018).

[23] N. Armesto and A. Capella, A Quantitative reanalysis of $J / \psi$ suppression in nuclear collisions, Phys. Lett. B 430, 23 (1998).

[24] R. Aaij et al. (LHCb Collaboration), Measurement of charged particle multiplicities and densities in $p p$ collisions at $\sqrt{s}=7 \mathrm{TeV}$ in the forward region, Eur. Phys. J. C 74, 2888 (2014).

[25] LHCb Collaboration, Multiplicity-dependent modification of $\chi_{c 1}(3872)$ and $\psi(2 S)$ production in $p p$ collisions at $\sqrt{s}=8 \mathrm{TeV}$, CERN Report No. LHCb-CONF-2019-005, 2019.

[26] S. Fleming, M. Kusunoki, T. Mehen, and U. van Kolck, Pion interactions in the $X(3872)$, Phys. Rev. D 76, 034006 (2007).

[27] E. Braaten, H. Hammer, and T. Mehen, Scattering of an ultrasoft pion and the $X(3872)$, Phys. Rev. D 82, 034018 (2010).

[28] E. Braaten, Galilean-invariant effective field theory for the X(3872), Phys. Rev. D 91, 114007 (2015).

[29] E. Braaten, L.-P. He, and J. Jiang, Galilean-invariant effective field theory for the $X(3872)$ at next-to-leading order, Phys. Rev. D 103, 036014 (2021).

[30] E. Braaten, L.-P. He, K. Ingles, and J. Jiang, Scattering of pions with $X(3872)$ (to be published).

[31] S. Cho and S. H. Lee, Hadronic effects on the X(3872) meson abundance in heavy ion collisions, Phys. Rev. C 88, 054901 (2013).

[32] L. M.Abreu, K. P. Khemchandani, A. Martinez Torres, F. S. Navarra, and M. Nielsen, X(3872) production and absorption in a hot hadron gas, Phys. Lett. B 761, 303 (2016).

[33] J. Hong, S. Cho, T. Song, and S. H. Lee, Hadronic effects on the $c c \bar{q} \bar{q}$ tetraquark state in relativistic heavy ion collisions, Phys. Rev. C 98, 014913 (2018).

[34] Z. w. Lin, T. G. Di, and C. M. Ko, Charm meson scattering cross-sections by pion and rho meson, Nucl. Phys. A689, 965 (2001).

[35] A. A. Alves, Jr. et al. (LHCb Collaboration), The LHCb detector at the LHC, J. Instrum. 3, S08005 (2008).

[36] CMS Collaboration, Evidence for $\chi_{c 1}(3872)$ in $\mathrm{PbPb}$ collisions and studies of its prompt production at $\sqrt{s_{\mathrm{NN}}}=$ 5.02 TeV, CERN Report No. CMS-PAS-HIN-19-005, 2019.s 